\title{
Impact of Electronic Physician Order-Set on Antibiotic Ordering Time in Septic Patients in the Emergency Department
}

\author{
Emily L. Fargo ${ }^{1}$ Frank D’Amico ${ }^{1}$ Aaron Pickering ${ }^{1}$ Kathleen Fowler ${ }^{2}$ Ronald Campbell ${ }^{1}$ \\ Megan Baumgartner ${ }^{1}$
}

${ }^{1}$ UPMC St. Margaret, Pittsburgh, Pennsylvania, United States

${ }^{2}$ Department of Pharmacy, UPMC Work Partners, Pittsburgh, Pennsylvania, United States Address for correspondence Emily L. Fargo, PharmD, BCPS,
UPMC St. Margaret, 815 Freeport Road, Pittsburgh, PA 15215
(e-mail: Elinville12@gmail.com).

Appl Clin Inform 2018;9:869-874.

\section{Abstract}

Keywords

- sepsis

- emergency medicine

- medical order entry systems

- quality of health care
Background Sepsis is a serious medical condition that can lead to organ dysfunction and death. Research shows that each hour delay in antibiotic administration increases mortality. The Surviving Sepsis Campaign Bundles created standards to assist in the timely treatment of patients with suspected sepsis to improve outcomes and reduce mortality.

Objective This article determines if the use of an electronic physician order-set decreases time to antibiotic ordering for patients with sepsis in the emergency department (ED).

Methods A retrospective chart review was performed on adult patients who presented to the ED of four community hospitals from May to July 2016. Patients with severe sepsis and/ or septic shock were included. Primary outcome was the difference in time to antibiotic ordering in patients whose physicians utilized the order-set versus those whose physicians did not. Secondary outcomes included differences in time to antibiotic administration, time to lactate test, hospital length of stay, and posthospitalization disposition. The institution's Quality Improvement Committee approved the project.

Results Forty-five of 123 patients (36.6\%) with sepsis had physicians who used the order-set. Order-set utilization reduced the mean time to ordering antibiotics by 20 minutes (99 minutes, 95\% confidence interval [CI]: 69-128 vs. 119 minutes, 95\% Cl: 91-147), but this finding was not statistically significant. Mean time to antibiotic administration ( 145 minutes, $95 \% \mathrm{Cl}: 108-181$ vs. 182 minutes, $95 \% \mathrm{Cl}: 125-239$ ) and median time to lactate tests ( 12 minutes, $95 \% \mathrm{Cl}: 0-20$ vs. 19 minutes, $95 \% \mathrm{Cl}: 8-34$ ), although in the direction of the hypotheses, were not significantly different.

Conclusion Utilization of the order-set was associated with a potentially clinically significant, but not statistically significant, reduced time to antibiotic ordering in patients with sepsis. Electronic order-sets are a promising tool to assist hospitals with meeting the Centers for Medicare and Medicaid Services core measure.

\section{Background and Significance}

Sepsis is a life-threatening condition that can lead to organ dysfunction and tissue hypoperfusion resulting from a dysregulated host response to an infection. It is a leading cause of morbidity and mortality in the United States and is associated with roughly 750,000 deaths annually. ${ }^{1}$ There is a strong body of evidence demonstrating that early identification and management of patients with suspected sepsis and septic shock significantly improves patient outcomes and reduces mortality. ${ }^{2-6}$ The 2016 Surviving Sepsis received

March 23, 2018

accepted after revision

October 11, 2018 (c) 2018 Georg Thieme Verlag KG Stuttgart · New York
DOI https://doi.org/

10.1055/s-0038-1676040. ISSN 1869-0327. 
Table 1 Surviving sepsis campaign 3-hour and 6-hour bundles ${ }^{3}$

\begin{tabular}{|c|c|}
\hline \multirow{4}{*}{$\begin{array}{l}\text { To be completed } \\
\text { within } 3 \text { hours of } \\
\text { time to } \\
\text { presentation }\end{array}$} & - Measure lactate level \\
\hline & $\begin{array}{l}\text { - Obtain blood cultures prior to } \\
\text { administration of antibiotics }\end{array}$ \\
\hline & - Administer broad spectrum antibiotics \\
\hline & $\begin{array}{l}\text { - Administer } 30 \mathrm{~mL} / \mathrm{kg} \text { crystalloid for } \\
\text { hypotension or lactate } \geq 4 \mathrm{mmol} / \mathrm{L}\end{array}$ \\
\hline \multirow{3}{*}{$\begin{array}{l}\text { To be completed } \\
\text { within } 6 \text { hours of } \\
\text { time to } \\
\text { presentation }\end{array}$} & $\begin{array}{l}\text { - Apply vasopressors (if needed) to } \\
\text { achieve MAP } \geq 65 \mathrm{~mm} \mathrm{Hg}\end{array}$ \\
\hline & $\begin{array}{l}\text { - Reassess fluid status if persistent } \\
\text { hypotension }\end{array}$ \\
\hline & $\begin{array}{l}\text { - Remeasure lactate if initial level was } \\
\text { elevated }\end{array}$ \\
\hline
\end{tabular}

Abbreviation: MAP, mean arterial pressure.

Campaign guidelines created two sets of standards known as the 3- and 6-hour "bundles" (-Table 1), consisting of elements of care that when implemented as a group, have been shown to improve patient outcomes. ${ }^{3}$ Items that must be completed within 3 hours of patient presentation to a health care facility (i.e., the "3-hour bundle") include: (1) measuring a lactate level; (2) obtaining blood cultures prior to administration of antibiotics; (3) administering broad spectrum antibiotics; and (4) administering $30 \mathrm{~mL} / \mathrm{kg}$ of crystalloids for hypotension or a lactate level of $\geq 4 \mathrm{mmol} / \mathrm{L}^{3}$ These guidelines strongly recommend the initiation of antimicrobials within 1 hour of severe sepsis, as research shows that each hour delay in antibiotic administration is associated with a $7.6 \%$ increase in mortality. ${ }^{3,7,8}$ In fact, in 2018 there was an update to the 2016 sepsis guidelines that recommended combining the 3-hour bundle and parts of the 6-hour bundle into a combined 1-hour bundle with the intention to expedite patient resuscitation and management. ${ }^{9}$ As of now, the 3-hour and 6-hour sepsis bundles are Centers for Medicare and Medicaid Services (CMS) core measures that are expected to influence future hospital reimbursement.

The emergency department (ED) is a main point of entry for patients with sepsis and therefore plays an integral role in assisting hospitals to meet the 3 -hour bundle criteria. ${ }^{10}$ Historically, health care institutions have struggled to promptly identify and treat patients with suspected sepsis. ${ }^{7}$ Multiple institutions have therefore begun utilizing technology to assist with the timely surveillance and treatment of patients with severe sepsis. ${ }^{10-18}$ One study showed that incorporating an electronic order-set for patients with suspected sepsis into their ED workflow resulted in decreased time to antibiotic administration, increased percentage of drawing two sets of blood cultures prior to administering antibiotics, and more appropriate antibiotic selection. ${ }^{10}$ In October 2015, the hospital system implemented an optional ED electronic physician order-set aimed to assist with the early identification and treatment of patients with suspected sepsis. All ED physicians within the health system were made aware of this order-set through ED provider meetings, email communications, and word of mouth. Since its initiation, there has been limited evaluation of the order-set to determine if it is helping to improve compliance with the 3-hour bundle criteria.

\section{Objective}

The goal of this project was to evaluate if the physician orderset improved time to sepsis treatment in the ED by comparing the time to broad-spectrum antibiotic ordering in patients whose physicians utilized the electronic order-set to those whose physicians did not utilize the order-set. It was hypothesized that physician utilization of the ED sepsis order-set results in a more rapid initiation of broad-spectrum antibiotics in patients with severe sepsis.

\section{Methods}

\section{Population}

A retrospective chart review was performed on four affiliated community hospitals around Pittsburgh, Pennsylvania, United States. All four hospitals had access to the same electronic physician order-set. Adults at least 18 years old who had International Classification of Diseases, 10th Edition (ICD-10) diagnosis codes (R65.20; R65.21) of severe sepsis (defined as sepsis with evidence of organ dysfunction) and/or septic shock (defined as sepsis with systolic blood pressure $<90 \mathrm{~mm} \mathrm{Hg}$ or lactate $\geq 4 \mathrm{mmol} / \mathrm{L}$ ) from May to July 2016 were included in the project ( - Fig. 1). Patients were excluded if they were not capable of meeting the CMS bundle criteria: (1) patients not admitted through the ED (e.g., direct admits, transfers, or patients with scheduled surgery) since there was no opportunity for the electronic physician order-set to be utilized; (2) if sepsis was due to infectious causes that would not require CMS-approved broad-spectrum antibiotics for empiric sepsis treatment, such as Clostridium difficile infection; and (3) if systemic inflammatory response syndrome(SIRS) criteria was not met during their hospitalization or if it was met greater than 3 hours after ED admission. The time that patients met SIRS criteria was defined as the time at which at least two of the four following criteria were met in the ED as documented in the electronic health record: heart rate (HR) $>90$ beats per minute $(\mathrm{bpm})$; respiratory rate $(\mathrm{RR})>20 \mathrm{bpm}$; white blood cell $>12,000$ or $<4,000 \times 10^{3} / \mu \mathrm{L}$; or temperature $>38.3^{\circ} \mathrm{C}$ or $<36^{\circ} \mathrm{C}$. Time to ED triage was defined as the earliest documented time of any vital recorded in the electronic health record. This project was submitted to the Institutional Review Board but was deemed to be a quality improvement project due to comparison of outcomes for patients within a single institution. Therefore, it was submitted to and approved by the hospital system's Quality Improvement Review Committee.

\section{Outcomes}

The primary outcome was the time from positive SIRS criteria to ordering of CMS-approved broad-spectrum antibiotics (-Appendix A). Time to ordering antibiotics was chosen 


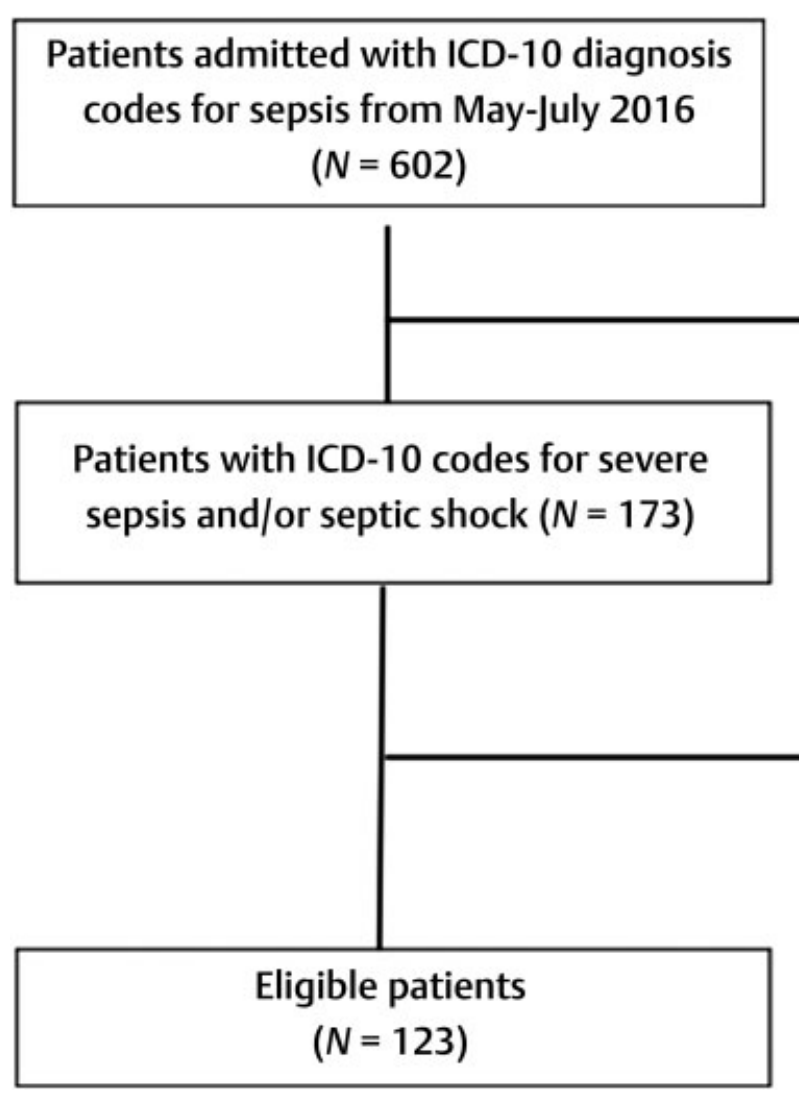

Fig. 1 Patient selection flow diagram.

instead of time to antibiotic administration because many extraneous factors, such as diagnostic testing and difficulties obtaining intravenous access, can potentially delay the administration time of antibiotics once they are ordered. Secondary outcomes included time from SIRS criteria to antibiotic administration, time from SIRS criteria to lactate test, hospital length of stay, and patient disposition upon discharge. Time of SIRS onset was used as time zero for the outcome intervals because the electronic health record does not document the time of ED presentation.

\section{Data Collection}

All data were collected via the inpatient electronic health record. For the primary and secondary outcomes, data that were retrieved included use of the electronic physician order-set, time of meeting SIRS criteria, time of ED triage, time of antibiotic ordering, time of antibiotic administration, time of lactate test, hospital length of stay, and patient disposition. SIRS criteria were collected to determine time of sepsis presentation instead of the quick Sepsis-Related Organ Failure Assessment (qSOFA) score because CMS has not yet adopted qSOFA for its reporting system. Other covariate data that were collected included patient age, sex, and time and date of ED admission.

\section{Statistical Analysis}

Basic descriptive statistics were used to describe the patient characteristics between the groups either receiving $(n=45)$

\section{Patients without ICD-10 diagnosis codes for severe sepsis or septic shock $(N=429)$ where excluded}

Table 2 Participant characteristics organized according to use of the electronic order-set

\begin{tabular}{|l|l|l|}
\hline \multirow{2}{*}{ Variable } & \multicolumn{2}{l|}{$\begin{array}{l}\text { Use of electronic order-set } \\
(N=123)\end{array}$} \\
\cline { 2 - 3 } & Yes $(\boldsymbol{n}=45)$ & No $(\boldsymbol{n}=78)$ \\
\hline Male sex (frequency, \%) & $21(47)$ & $32(41)$ \\
\hline Age, y (mean, SD) & $74.8 \pm 15.3$ & $69.3 \pm 15.7$ \\
\hline \multicolumn{2}{|l|}{ SIRS criteria met (frequency, \%) } \\
\hline HR, RR & $21(47)$ & $43(55)$ \\
\hline HR, WBC & $6(13)$ & $18(23)$ \\
\hline HR, Temperature & $10(22)$ & $9(12)$ \\
\hline All other combinations & $8(18)$ & $8(10)$ \\
\hline ED admission time (frequency, \%) \\
\hline 00:00-08:00 & $11(25)$ & $11(14)$ \\
\hline 08:00-16:00 & $21(47)$ & $28(36)$ \\
\hline 16:00-00:00 & $13(29)$ & $39(50)$ \\
\hline
\end{tabular}

Abbreviations: ED, emergency department; HR, heart rate; RR, respiratory rate; SD, standard deviation; SIRS, systemic inflammatory response syndrome; WBC, white blood cell.

Note: Data represents frequency and percentage unless otherwise specified.

SIRS criteria: HR (heart rate) $>90$ beats per minute (bpm), RR (respiratory rate) $>20 \mathrm{bpm}, \mathrm{WBC}>12,000$ or $<4,000$, temperature $>38.3^{\circ} \mathrm{C}$ or $<36^{\circ} \mathrm{C}$. 
or not receiving $(n=78)$ the electronic order-set. For continuous variables, means were computed if data were normally distributed, otherwise medians and interquartile ranges were used if data were skewed. Either parametric or nonparametric statistical tests were used to univariately compare patient characteristics between the two groups. Results of these univariate tests showed no statistical differences at $p>0.05$. However, age, SIRS criteria met, and admission time had $p$-values less than 0.20 and these were still entered into separate multiple regression models for each time outcome. Standard errors estimated from the multiple regression models were used to construct the $95 \%$ confidence intervals (CIs). If two 95\% individual Cls overlapped, then results would be considered not statistically significant $(p>0.05)$.

Eight cases were omitted because they did not meet the inclusion criteria for SIRS being met within 3 hours of ED presentation. The patient characteristics between the 8 excluded and the remaining 123 included patients were compared for differences in patient characteristics. For all the patient characteristics (except use of the order-set), the 8 cases were approximately evenly distributed. Regarding use of the order-set, 7 of the 8 cases did not get the order-set and no further analysis was done.

\section{Results}

There were 602 patients admitted with an ICD-10 diagnosis code for sepsis in May, June, and July 2016, and 131 were eligible for chart review after accounting for exclusion criteria ( - Fig. 1). The overall mean values for time of SIRS criteria to antibiotic order and administration were imputed for the missing data in one case because a broad-spectrum antibiotic was never ordered. It was decided to include this individual in the analysis to maintain the "intention to treat" principle by not subjectively removing any cases. Because utilization of the order-set was not mandatory, multiple regression analyses were used to estimate adjusted mean times to ordering. Physicians utilized the order-set in 45 (36\%) of the patients who were admitted through the ED with suspected sepsis ( - Table 2). A majority of the patients included were female (59.5\%) and 101 patients (77\%) presented to the ED between 8 AM and 12 Am. The most common combination of two SIRS criteria met was HR $>90 \mathrm{bpm}$ and RR $>20 \mathrm{bpm}$. Out of the 131 patient charts reviewed, 45 (34.4\%) patients had ED physicians who utilized the electronic order-set.

Patients whose physicians utilized the order-set were ordered broad-spectrum antibiotics on average 20 minutes sooner than patients whose physicians did not utilize the order-set (98.9 minutes, $95 \%$ CI: $69.4-128.4$ vs. 119.1 minutes, 95\% CI: 91.4-146.7); however, this difference was not found to be statistically significant $(p>0.05)$. Use of the order-set resulted in reduced mean time to antibiotic administration (144.5 minutes, 95\% CI: 107.5-181.4 vs. 182.4 minutes, 95\% CI: 125.8-239.0) and reduced median time to lactate test (12 minutes, range: $0-20$ vs. 18.5 minutes, range: $8-34)$, similarly the differences were not statistically significant $(p>0.05)$. Cls based on the median were used to compare time to lactate test because roughly $25 \%$ of lactate tests were drawn prior to SIRS criteria being met, which would have resulted in a negative mean time. Patients who had the order-set utilized were found to have longer hospital lengths of stay (8.4 vs. 7.3 days). There was no difference $(p>0.05)$ in patient disposition upon discharge between the two groups. Results of multivariate analyses did not show statistical significance between the groups for either the time from SIRS criteria to antibiotic ordering, antibiotic administration, or time from triage. The means and CIs are all shown in -Table 3.

\section{Discussion}

In this multicenter retrospective chart review, we found that the use of the ED electronic order-set did not show a significant reduction in time in either antibiotic ordering ( $\sim 20$ minutes) or administration ( $\sim 38$ minutes). Patients treated with the order-set had a mean time to antibiotic administration of 144.5 minutes (95\% CI: $107.5-181.4$ ) compared with 182.4 minutes (95\% CI: 125.8-239.0) without the order-set. Regardless of the lack of statistical significance, the electronic order-set did reduce the time to antibiotic administration in patients with suspected sepsis, and the results suggest that the utilization of this order-set may help hospitals meet the CMS core measure of administering antibiotics within 3 hours (180 minutes) of sepsis presentation, but further research is required.

Strengths of this project include it being multicenter and having objective outcome measures. Limitations include it being retrospective, having a high risk of selection bias due to the order-set being optional, low physician utilization of the order-set, and the lack of generalizability to other hospitals since the order-set is specific to only one health system. Another major limitation was the small sample size, which could have influenced the results showing nonstatistically significant changes. There were also numerous confounders that could have potentially affected the results, such as patient acuity, voluntary use of the order-set by physicians, and physician comfort level with using electronic order-sets. Patients who had the order-set utilized on average had a 1day longer length of stay, suggesting that physicians may have preferentially chosen to utilize the order-set in higher acuity patients. Another potential confounder is the design of the order-set. Generally, assessment and management of sepsis is divided into two phases: (1) patient work-up (checking vitals, ordering laboratories and cultures, etc.) and (2) treatment (fluid resuscitation, antibiotic selection, etc.). This specific order-set is monophasic, in which physicians are prompted to order all the laboratories required for patient assessment at the same time as being prompted to order antibiotics and fluids. As a result, it is possible that physicians are initially utilizing the order-set for the recommended laboratories and cultures, but may not be returning to the order-set once it is time to order antibiotics. This could result in extended time to antibiotic ordering.

Overall, this review demonstrates that the use of electronic order-sets in the ED has the potential to reduce both time 
Table 3 Outcomes organized according to use of the electronic order-set

\begin{tabular}{|c|c|c|c|c|}
\hline \multirow[t]{3}{*}{ Outcomes } & \multicolumn{4}{|c|}{ Use of electronic order-set $(N=123)$} \\
\hline & \multicolumn{2}{|c|}{ Yes $(N=45)$} & \multicolumn{2}{|c|}{ No $(N=78)$} \\
\hline & Mean & $95 \% \mathrm{Cl}$ & Mean & $95 \% \mathrm{Cl}$ \\
\hline SIRS to antibiotic order, minutes ${ }^{\mathrm{a}}$ & 98.9 & $(69.4-128.4)$ & 119.1 & $(91.4-146.7)$ \\
\hline $\begin{array}{l}\text { SIRS to antibiotic } \\
\text { administration, minutes }^{\text {a }}\end{array}$ & 144.5 & $(107.5-181.4)$ & 182.4 & $(125.8-239.0)$ \\
\hline Triage to antibiotic order, minutes ${ }^{\mathrm{a}}$ & 113.7 & $(80.4-146.9)$ & 145.9 & $\begin{array}{l}(120.9-170.8) \\
\end{array}$ \\
\hline $\begin{array}{l}\text { SIRS to lactate level, min }{ }^{\text {b }} \text { (median, 95\% } \\
\mathrm{CI} \text { for median) }\end{array}$ & 12 & $0-20$ & 18.5 & $8-34$ \\
\hline Length of stay, days ${ }^{a}$ & 8.4 & $(5.7-11.0)$ & 7.3 & $(6.1-8.4)$ \\
\hline \multicolumn{5}{|l|}{ Disposition (frequency, \%) } \\
\hline Home & $11(24)$ & & $26(33)$ & \\
\hline Skilled nursing facility or rehabilitation & $22(49)$ & & $34(44)$ & \\
\hline Death & $12(27)$ & & $18(23)$ & \\
\hline
\end{tabular}

Abbreviations: $\mathrm{Cl}$, confidence interval; SIRS, systemic inflammatory response syndrome.

${ }^{a}$ Mean values and their confidence intervals represent the least squares estimates from the multiple regression models, adjusted for age, SIRS criteria, and admission time.

${ }^{\mathrm{b}}$ Data represent the median values displayed. Exact statistical methods were used to obtain the confidence intervals.

to antibiotic ordering and administration in patients with suspected sepsis, and potentially improve hospitals' compliance with meeting the Sepsis CMS Core Measures.

\section{Conclusion}

The use of the electronic order-set was associated with reduced time to antibiotic ordering and administration in patients with suspected sepsis in the ED; however, the findings were not statistically significant.

\section{Clinical Relevance Statement}

This project looked at the efficacy of an electronic tool that may be used to help improve patient outcomes in addition to helping the hospital system meet the Sepsis CMS Core Measure. Even though this specific order-set did not show statistically improved time to antibiotic ordering and administration in patients with severe sepsis, electronic order-sets in the ED have great potential to help hospital systems meet the Sepsis CMS Core criteria 3-hour bundle. Low utilization of the sepsis order-set by physicians may require further investigation.

\section{Multiple Choice Questions}

1. When implementing an electronic physician order-set in the emergency department, which of the following is more important to take into consideration when designing the order-set?
a. Physician input.
b. Nursing input.
c. eRecord input.
d. Administration input.

Correct Answer: The correct answer is option a, physician input. Since the physician will be the only provider utilizing the order-set, it is important that they provide input to assist in the design of the order-set so that it matches their workflow. This may also encourage more physicians to utilize the order-set since they would assist with the design.

2. Which of the following is a criterion for the Surviving Sepsis Campaign 3-hour bundle?

a. Measure procalcitonin level.

b. Administer broad spectrum antibiotics.

c. Obtain blood cultures after administering antibiotics.

d. Administer albumin replacement for hypotension and resuscitation.

Correct Answer: The correct answer is option b, Administer broad spectrum antibiotics. Administration of broad spectrum antibiotics is one of the 3-hour bundle criteria, that is now being used by CMS as a quality member that influences hospital reimbursement for patients admitted with sepsis. The other criteria of the 3-hour bundle are: (1) measure lactate level; (2) obtaining blood cultures prior to administration of antibiotics; and (3) administer $30 \mathrm{~mL} / \mathrm{kg}$ of crystalloid solution (albumin is a colloid) for hypotension or lactate $\geq 4 \mathrm{mmol} / \mathrm{L}$.

3. What is the most important reason for developing an electronic order-set to be used in the emergency department to aid in the management of patients with sepsis?

a. Improve hospital reimbursement rates.

b. Physician satisfaction.

c. Reduce hospital costs.

d. Improve patient safety and outcomes. 
Correct Answer: The correct answer is option d, Improve patient safety and outcomes. Everything in health care should be done with the primary focus of improving patient care. Reducing health care spending and optimizing hospital reimbursement is very important, but the patient should always be the first priority.

\section{Protection of Human and Animal Subjects}

This project was a retrospective chart review and was exempt from the Institutional Review Board.

\section{Conflict of Interest}

None declared.

\section{Acknowledgments}

We acknowledge the institution's Quality Improvement Committee for allowing us to pursue this project to help understand the utility of the electronic order-set. We also thank the hospital librarian for assisting with the literature search for our background section.

\section{References}

1 Gaieski DF, Edwards JM, Kallan MJ, Carr BG. Benchmarking the incidence and mortality of severe sepsis in the United States. Crit Care Med 2013;41(05):1167-1174

2 Kumar A, Zarychanski R, Light B, et al; Cooperative Antimicrobial Therapy of Septic Shock (CATSS) Database Research Group. Early combination antibiotic therapy yields improved survival compared with monotherapy in septic shock: a propensity-matched analysis. Crit Care Med 2010;38(09):1773-1785

3 Rhodes A, Evans LE, Alhazzani W, et al. Surviving Sepsis Campaign: International Guidelines for Management of Sepsis and Septic Shock: 2016. Crit Care Med 2017;45(03):486-552

4 Rivers E, Nguyen B, Havstad S, et al; Early Goal-Directed Therapy Collaborative Group. Early goal-directed therapy in the treatment of severe sepsis and septic shock. N Engl J Med 2001;345(19): $1368-1377$

5 Levy MM, Rhodes A, Phillips GS, et al. Surviving Sepsis Campaign: association between performance metrics and outcomes in a 7.5year study. Intensive Care Med 2014;40(11):1623-1633

Appendix A List of common CMS-approved broad-spectrum antibiotics for empiric monotherapy of sepsis ${ }^{19}$

\begin{tabular}{|l|l|}
\hline Antibiotic class & $\begin{array}{l}\text { Approved antibiotics for } \\
\text { monotherapy }\end{array}$ \\
\hline \multirow{2}{*}{ Penicillins } & Piperacillin/tazobactam \\
\cline { 2 - 2 } & Ampicillin/sulbactam \\
\hline \multirow{3}{*}{ Cephalosporins } & Ceftriaxone \\
\cline { 2 - 2 } & Cefepime \\
\cline { 2 - 2 } & Ceftaroline \\
\hline \multirow{2}{*}{ Carbapenems } & Meropenem \\
\cline { 2 - 2 } & Ertapenem \\
\hline Fluoroquinolones & Levofloxacin \\
\hline
\end{tabular}

Abbreviation: CMS, Centers for Medicare and Medicaid Services.
6 Gao F, Melody T, Daniels DF, Giles S, Fox S. The impact of compliance with 6-hour and 24-hour sepsis bundles on hospital mortality in patients with severe sepsis: a prospective observational study. Crit Care 2005;9(06):R764-R770

7 Ferrer R, Martin-Loeches I, Phillips G, et al. Empiric antibiotic treatment reduces mortality in severe sepsis and septic shock from the first hour: results from a guideline-based performance improvement program. Crit Care Med 2014;42 (08):1749-1755

8 Kumar A, Roberts D, Wood KE, et al. Duration of hypotension before initiation of effective antimicrobial therapy is the critical determinant of survival in human septic shock. Crit Care Med 2006;34(06):1589-1596

9 Levy MM, Evans LE, Rhodes A. The Surviving Sepsis Campaign Bundle: 2018 update. Crit Care Med 2018;46(06):997-1000

10 Idrees M, Macdonald SP, Kodali K. Sepsis Early Alert Tool: early recognition and timely management in the emergency department. Emerg Med Australas 2016;28(04):399-403

11 Paul M, Andreassen S, Nielsen AD, et al; TREAT Study Group. Prediction of bacteremia using TREAT, a computerized decision-support system. Clin Infect Dis 2006;42(09):1274-1282

12 Harrison AM, Herasevich V, Gajic O. Automated sepsis detection, alert, and clinical decision support: act on it or silence the alarm? Crit Care Med 2015;43(08):1776-1777

13 Henry KE, Hager DN, Pronovost PJ, Saria S. A targeted real-time early warning score (TREWScore) for septic shock. Sci Transl Med 2015;7(299):299ra122

14 Nguyen SQ, Mwakalindile E, Booth JS, et al. Automated electronic medical record sepsis detection in the emergency department. PeerJ 2014;2:e343

15 Bailey TC, Chen Y, Mao Y, et al. A trial of a real-time alert for clinical deterioration in patients hospitalized on general medical wards. J Hosp Med 2013;8(05):236-242

16 Semler MW, Weavind L, Hooper MH, et al. An electronic tool for the evaluation and treatment of sepsis in the ICU: a randomized controlled trial. Crit Care Med 2015;43(08):1595-1602

17 Nelson JL, Smith BL, Jared JD, Younger JG. Prospective trial of realtime electronic surveillance to expedite early care of severe sepsis. Ann Emerg Med 2011;57(05):500-504

18 Buck KM. Developing an early sepsis alert program. J Nurs Care Qual 2014;29(02):124-132

19 Specifications Manual for National Hospital Inpatient Quality Measures. Discharges 07-01-2016 (3Q16) through 12-31-16 (4Q16). Version 5.1. Appendix C-2. Table 5.0: Antibiotic monotherapy, sepsis 